NASA/TM-2003-212461

\title{
The Role of Instability Waves in Predicting Jet Noise
}

Marvin E. Goldstein and Louis M. Handler

Glenn Research Center, Cleveland, Ohio 
Since its founding, NASA has been dedicated to the advancement of aeronautics and space science. The NASA Scientific and Technical Information (STI) Program Office plays a key part in helping NASA maintain this important role.

The NASA STI Program Office is operated by Langley Research Center, the Lead Center for NASA's scientific and technical information. The NASA STI Program Office provides access to the NASA STI Database, the largest collection of aeronautical and space science STI in the world. The Program Office is also NASA's institutional mechanism for disseminating the results of its research and development activities. These results are published by NASA in the NASA STI Report Series, which includes the following report types:

- $\quad$ TECHNICAL PUBLICATION. Reports of completed research or a major significant phase of research that present the results of NASA programs and include extensive data or theoretical analysis. Includes compilations of significant scientific and technical data and information deemed to be of continuing reference value. NASA's counterpart of peerreviewed formal professional papers but has less stringent limitations on manuscript length and extent of graphic presentations.

- TECHNICAL MEMORANDUM. Scientific and technical findings that are preliminary or of specialized interest, e.g., quick release reports, working papers, and bibliographies that contain minimal annotation. Does not contain extensive analysis.

- CONTRACTOR REPORT. Scientific and technical findings by NASA-sponsored contractors and grantees.
- CONFERENCE PUBLICATION. Collected papers from scientific and technical conferences, symposia, seminars, or other meetings sponsored or cosponsored by NASA.

- SPECIAL PUBLICATION. Scientific, technical, or historical information from NASA programs, projects, and missions, often concerned with subjects having substantial public interest.

- TECHNICAL TRANSLATION. Englishlanguage translations of foreign scientific and technical material pertinent to NASA's mission.

Specialized services that complement the STI Program Office's diverse offerings include creating custom thesauri, building customized databases, organizing and publishing research results ... even providing videos.

For more information about the NASA STI Program Office, see the following:

- Access the NASA STI Program Home Page at http://www.sti.nasa.gov

- E-mail your question via the Internet to help@sti.nasa.gov

- Fax your question to the NASA Access Help Desk at 301-621-0134

- Telephone the NASA Access Help Desk at 301-621-0390

- Write to:

NASA Access Help Desk

NASA Center for AeroSpace Information 7121 Standard Drive

Hanover, MD 21076 
NASA/TM-2003-212461

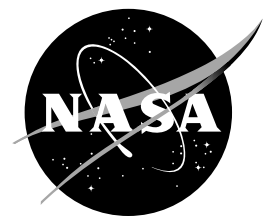

\section{The Role of Instability Waves in Predicting Jet Noise}

Marvin E. Goldstein and Louis M. Handler

Glenn Research Center, Cleveland, Ohio

Prepared for the

Ninth Aeroacoustics Conference and Exhibit cosponsored by the American Institute of Aeronautics and Astronautics and the Confederation of European Aerospace Societies

Hilton Head, South Carolina, May 12-14, 2003

National Aeronautics and

Space Administration

Glenn Research Center 


\section{Acknowledgments}

The authors would like to thank Dr. Lennart Hultgren for supplying the Rayleigh equation eigenvalue code and Dr. Stewart Leib for pointing out an error.

This report contains preliminary

findings, subject to revision as analysis proceeds.

Available from

NASA Center for Aerospace Information 7121 Standard Drive

Hanover, MD 21076
National Technical Information Service 5285 Port Royal Road Springfield, VA 22100 


\title{
THE ROLE OF INSTABILITY WAVES IN PREDICTING JET NOISE
}

\author{
M.E. Goldstein ${ }^{*}$ and L.M. Handler ${ }^{\dagger}$ \\ National Aeronautics and Space Administration \\ Glenn Research Center \\ Cleveland, Ohio 44135
}

\begin{abstract}
$\underline{\text { Abstract }}$
Debate over whether linear instability waves play a role in the prediction of jet noise has been going on for many years. Parallel mean flow models, such as the one proposed by Lilley, ${ }^{2}$ usually neglect these waves because they cause the solution to become infinite. The present paper solves the true non-parallel acoustic equations for a two-dimensional shear layer by using a vector Greens function and assuming small mean flow spread rate. The results show that linear instability waves must be accounted for in order to construct a proper causal solution to the problem.

\section{$\underline{\text { Introduction }}$}

Current industrial noise prediction methods, such as the MGB approach, ${ }^{1}$ are based on Lilley's ${ }^{2}$ equation which involves a wave operator appropriate to sound propagation on a parallel mean flow. This equation possesses homogeneous solutions corresponding to spatially growing instability waves on that flow and its complete solution consists of a particular solution plus these homogeneous contributions. But the result is meaningless because the instability waves become unbounded (infinite) far downstream in the flow. The usual resolution to this dilemma is to completely neglect the contribution of the instability waves. Dowling, et al. ${ }^{3}$ attempted to justify this by invoking causality arguments.
\end{abstract}

A better approach is to begin with an equation appropriate to sound propagation on a non-parallel flow, say the actual mean flow in the jet. The most important difference between this approach and Lilley's parallel flow result is that the homogeneous solutions to the acoustic equations correspond to instability waves that grow and then decay on the diverging non-parallel base flow and therefore always remain bounded, which eliminates the dilemma alluded to above.

A vector Greens function approach is used in reference 4 to obtain a general solution to the non-parallel acoustic equations by assuming that the spread rate of the mean flow is small. The relevant Green's function, which must satisfy an appropriate causality condition, consists of two components - both of which act on the

\footnotetext{
${ }^{*}$ Chief Scientist, Fellow AIAA

${ }^{\dagger}$ Computer Scientist
}

same source term. The first corresponds to the usual Lilley equation solution but with slightly modified source terms. The second is associated with linear instability waves but is very different from conventional instability models since these waves are now continuously generated along the length of the jet and do not constitute separate sound sources. They merely serve as conduits to carry the sound to the far field.

The Greens Function can be thought of as a filter that only allows certain parts of the source to radiate. Each of its two components responds to different portions of the source spectrum causing the radiation field to exhibit a bi-model structure. The contribution of the second Greens function component is fairly small at subsonic Mach numbers but can be significant at supersonic speeds. The result is therefore somewhat different from the conventional parallel flow models.

The present paper applies the general result of reference 4 to a two-dimensional shear layer with fully developed similarity form, which greatly simplifies the final equations.

\section{$\underline{\text { The Fundamental Equations }}$}

We let

$$
h_{o} \equiv h+\frac{1}{2} v^{2}
$$

denote the stagnation enthalpy, $h$ the enthalpy, $t$ the time, $\boldsymbol{x} \equiv\left\{x_{1}, x_{2}, x_{3}\right\}$ the Cartesian coordinates, $p$ the pressure, $\rho$ the density, $\boldsymbol{v}=\left\{v_{1}, v_{2}, v_{3}\right\}$ the fluid velocity, $\sigma_{i j}$ the viscous stress tensor, and $q_{i}$ the heat flux vector. The flow variables are assumed to satisfy the ideal gas law

$$
p=\rho R T, \quad h=c_{p} T
$$

with $R=c_{p}-c_{v}$ being the gas constant, $c_{p}$ and $c_{v}$ the specific heats at constant pressure and volume and $T$ the absolute temperature.

References 5 and 19 show that the Navier-Stokes equations can be recast into the convective form of the linearized Navier-Stokes equations by dividing the dependent variables

$$
\rho=\bar{\rho}+\rho^{\prime}, \quad p=\bar{p}+p^{\prime}, \quad h=\tilde{h}+h^{\prime}, \quad v_{i}=\tilde{v}_{i}+v_{i}^{\prime}
$$


as well as the viscous terms $\sigma_{i j}$ and $q_{i}$, into their 'base flow' components $\bar{\rho}, \bar{p}, \tilde{h}, \tilde{v}_{i}, \bar{\sigma}_{i j}$, and $\bar{q}_{i}$ and into their 'residual' components $\rho^{\prime}, p^{\prime}, h^{\prime}, v_{i}^{\prime}, \sigma_{i j}^{\prime}$, and $q_{i}^{\prime}$ and requiring that the former satisfy the inhomogeneous Navier-Stokes equations

$$
\begin{gathered}
\frac{D_{o}}{D t} \bar{\rho}=0 \\
\frac{D_{o}}{D t} \bar{\rho} \tilde{v}_{i}+\frac{\partial \bar{p}}{\partial x_{i}}=\frac{\partial}{\partial x_{j}}\left(\tilde{T}_{i j}+\bar{\sigma}_{i j}\right) \\
\frac{D_{o}}{D t} \bar{\rho} \tilde{h}_{o}-\frac{\partial \bar{p}}{\partial t}=\frac{D_{o} \tilde{H}_{o}}{D t}+\frac{\partial}{\partial x_{j}}\left(\tilde{H}_{j}-\tilde{q}_{j}+\tilde{v}_{i} \bar{\sigma}_{i j}\right)
\end{gathered}
$$

along with an ideal gas law equation of state,

$$
\tilde{h}=c_{p} \tilde{T}=\frac{c_{p}}{R} \frac{\bar{p}}{\bar{\rho}}
$$

where the operator $D_{o} / D t$ is not the usual convective derivative but is defined by

$$
\frac{D_{o}}{D t} f \equiv \frac{\partial f}{\partial t}+\frac{\partial}{\partial x_{j}}\left(\tilde{v}_{j} f\right)
$$

for any function $f$,

$$
\tilde{h}_{o} \equiv \tilde{h}+\frac{1}{2} \tilde{v}^{2}
$$

is the base flow stagnation enthalpy, and the 'sources strengths' $\tilde{T}_{i j}, \tilde{H}_{o}$, and $\tilde{H}_{j}$, which are assumed to be localized, can be arbitrarily specified.

The residual variables are governed by the convective form of the linearized Navier-Stokes (LNS) equations

$$
L_{\mu v} u_{v}=s_{\mu}, \quad \mu, v=1,2, \ldots 5
$$

where the linear operator $L_{\mu v}$, which is exactly the same as the operator that would be obtained by linearizing the Euler equations about the base flow (4), is written out in full in reference 5 . The Greek indices range from 1 to 5 , the Latin indices from 1 to 3 , the Einstein summation convention is being used, the fivedimensional dependent variable vector $u_{v}$ is given by

$$
\left\{u_{v}\right\} \equiv\left\{\rho v_{v}^{\prime}, \frac{p_{o}^{\prime}}{\gamma-1}, \quad \rho^{\prime}\right\}
$$

and the source term $s_{\mu}$ is given by

$s_{\mu} \equiv \frac{\partial}{\partial x_{j}}\left(e_{\mu j}^{\prime}-\tilde{e}_{\mu j}\right)+\delta_{\mu 4}\left(e_{i j}^{\prime}-\tilde{e}_{i j}\right) \frac{\partial \tilde{v}_{i}}{\partial x_{j}}$ for $\mu=1, \ldots 4$ where

$$
p_{o}^{\prime} \equiv p^{\prime}+\frac{\gamma-1}{2} \rho v^{\prime 2}+(\gamma-1) \tilde{H}_{o}
$$

The source strengths $e_{i v}^{\prime}-\tilde{e}_{i v}$ are given by

$$
\begin{gathered}
e_{i j}^{\prime} \equiv-\rho v_{i}^{\prime} v_{j}^{\prime}+\frac{\gamma-1}{2} \delta_{i j} \rho v^{\prime 2}+\sigma_{i j}^{\prime}, e_{i 4}^{\prime} \equiv-\rho v_{i}^{\prime} h_{o}^{\prime}-q_{i}^{\prime}+\sigma_{i j} v_{j}^{\prime} \\
\tilde{e}_{i j} \equiv \tilde{T}_{i j}-\delta_{i j}(\gamma-1) \tilde{H}_{o}, \tilde{e}_{i 4} \equiv \tilde{H}_{i}-\tilde{T}_{i j} \tilde{v}_{j}
\end{gathered}
$$

where

$$
h_{o}^{\prime}=h^{\prime}+\frac{1}{2} v^{\prime 2}
$$

Equations (8) are exact consequences of the original Navier-Stokes equations, but they have been rearranged so that their left-hand sides are the same as the equations that would have been obtained by linearizing the convective form of the Euler equations about the base flow (4). In other words, they are just the LNS equations with modified dependent variables and with the viscous stress perturbation replaced by the generalized Reynolds stress $e_{i j}^{\prime}-\tilde{e}_{i j}$ and the heat flux perturbation replaced by the generalized stagnation enthalpy flux $e_{i 4}^{\prime}-\tilde{e}_{i 4}$.In the acoustic analogy approach the right hand sides would be treated as known source terms and the linearized Euler operators $L_{\mu \nu}$ would be inverted to solve for the dependent variables. The fact that they are non-linear causes no particular difficulty since one of them, $p_{o}^{\prime}$, reduces to the linear pressure fluctuation in the far field.

The base flow equations (4) reduce to the usual Euler equations when the arbitrary source strengths $\tilde{T}_{i j}, \tilde{H}_{j}$, and $\tilde{H}_{o}$ and viscous terms $\bar{\sigma}_{i j}$ and $\bar{q}_{i}$ are set equal to zero. A general class of solutions to these equations, which conveniently provide good approximations to the actual mean flow fields in jets and shear layers, is the unidirectional transversely sheared mean flows

$$
\tilde{v}_{i}=\delta_{i 1} U\left(x_{2}, x_{3}\right), \bar{p}=\text { constant }, \quad \bar{\rho}=\bar{\rho}\left(x_{2}, x_{3}\right)
$$

The fifth equation (8) then decouples from the remaining four which become the inhomogeneous compressible Rayleigh equations ${ }^{6}$

$$
\begin{gathered}
\bar{\rho}\left(\frac{D_{o} u_{i}^{\prime}}{D t}+\delta_{i 1} u_{j}^{\prime} \frac{\partial U}{\partial x_{j}}\right)+\frac{\partial p_{o}^{\prime}}{\partial x_{i}}=\frac{\partial}{\partial x_{j}} e_{i j}^{\prime} \\
\frac{D_{o} p_{o}^{\prime}}{D t}+\gamma \bar{p} \frac{\partial u_{j}^{\prime}}{\partial x_{j}}=(\gamma-1)\left(\frac{\partial e_{4 j}^{\prime}}{\partial x_{j}}+e_{1 j}^{\prime} \frac{\partial U}{\partial x_{j}}\right)
\end{gathered}
$$


where $u_{i}^{\prime} \equiv u_{i} / \bar{\rho}$ and $D_{o} / \mathrm{Dt}$ now reduces to the usual convective derivative

$$
\frac{D_{o}}{D t}=\frac{\partial}{\partial t}+U \frac{\partial}{\partial x_{1}}
$$

It is well known (see chapter 1 of ref. 7) that the velocity-like variables $u_{i}^{\prime}$ can be eliminated between these equations (by taking the divergence of the first equation and the convective derivative of the second, subtracting the results and then using the first equation to eliminate the velocity fluctuation on the left-hand side) to obtain the Lilley ${ }^{2}$ equation

$$
L p_{o}^{\prime} \equiv s
$$

where

$$
L \equiv \frac{D_{o}}{D t}\left(\frac{\partial}{\partial x_{i}} \tilde{c}^{2} \frac{\partial}{\partial x_{i}}-\frac{\bar{D}^{2}}{D t^{2}}\right)-2 \frac{\partial U}{\partial x_{j}} \frac{\partial}{\partial x_{1}} \widetilde{c}^{2} \frac{\partial}{\partial x_{j}}
$$

is the variable density Pridmore-Brown ${ }^{8}$ operator,

$$
\tilde{c}^{2}=\gamma \bar{p} / \bar{\rho}\left(x_{2}, x_{3}\right)
$$

is the square of the mean flow sound speed and the source term $\mathrm{s}$ is written out in reference 5 . As noted in the Introduction, it possesses homogeneous solutions corresponding to spatially growing instability waves on the base flow (15). The complete solution to these equations consists of a particular solution plus contributions from these homogeneous solutions, but the result is meaningless because the instability waves become unbounded far downstream in the flow causing the solution to become infinite there. The usual resolution is to completely neglect the instability wave contribution.

A better resolution is to choose the base flow to be the actual mean flow of the jet. The over bars in the base flow equations then denotes the time average

$$
\bar{\bullet} \equiv \lim _{T \rightarrow \infty} \frac{1}{2 T} \int_{-T}^{T} \bullet(\mathbf{x}, t) \mathrm{d} t
$$

where the dot is a place holder for $\rho, v_{i}, p$, and $h$, and

$$
\tilde{\bullet} \equiv(\bar{\rho} \bullet / \bar{\rho})
$$

denotes a Favre averaged quantity ${ }^{8}$ for all variables except $\tilde{h}_{o}$, which is defined by Eq. (7). Notice that Eq. (20) is completely consistent with the overall ideal gas law (2) when the tilde is defined in this fashion.

The time derivatives drop out of the base flow equations and their source strengths are given by

$$
\begin{gathered}
\tilde{T}_{i j}=-\bar{\rho} \widetilde{v_{i}^{\prime} v_{j}^{\prime}} \\
\tilde{H}_{o}=\frac{1}{2} \tilde{T}_{i i} \\
\tilde{e}_{4 i} \equiv \tilde{H}_{i}-\tilde{T}_{i j} \tilde{v}_{j}=-\bar{\rho} \widetilde{h_{o}^{\prime}} v_{i}^{\prime}
\end{gathered}
$$

They are now the ordinary RANS equations, which do not, of course, form a closed system. They are usually closed by assuming some sort of model relating the source terms to the mean flow variables $\tilde{v}_{i}, \bar{\rho}, \bar{p}$, and $\tilde{h}$ and their derivatives, such as Boussinesq model ${ }^{8,9}$ for the Reynolds stresses and a similar model for $\tilde{H}_{j}$.

The most important difference between these results and the parallel flow result is that the homogeneous solutions to the LNS equations, which now correspond to instability waves growing and then decaying on the diverging non-parallel base flow, will always remain bounded. This eliminates the paradox alluded to above and the corresponding LNS equations can be used to calculate the radiated sound. The relevant solution consists of a particular solution that is driven by the sources (i.e. it satisfies causality and therefore the appropriate upstream boundary conditions) and a homogeneous solution that is driven by the upstream boundary conditions, say at the nozzle exit.

\section{The Green's Function Solution}

The particular solution that satisfies the appropriate outgoing wave boundary conditions can be written in terms of the causal vector Greens function for these equations $g_{v \sigma}\left(\boldsymbol{x}, t \mid \boldsymbol{x}^{\prime}, t^{\prime}\right)$, which satisfies ${ }^{11}$

$$
L_{\mu \nu} g_{v \sigma}=\delta_{\mu \sigma} \delta\left(\boldsymbol{x}-\boldsymbol{x}^{\prime}\right) \delta\left(t-t^{\prime}\right)
$$

and leads to the following formula for $u_{v}$

$$
u_{v}(\boldsymbol{x}, t)=\int_{-\infty}^{\infty} \int_{V} g_{v \mu}\left(\boldsymbol{x}, t \mid \boldsymbol{x}^{\prime}, t^{\prime}\right) s_{\mu}\left(\boldsymbol{x}^{\prime}, t^{\prime}\right) d \boldsymbol{x}^{\prime} d t^{\prime}
$$

where the symbol $V$ denotes integration over all space.

The derivatives can be transferred to the Greens function (see reference 4) to obtain

$$
p_{0}^{\prime}=\int_{-\infty}^{\infty} \int_{V} \gamma_{j \mu}\left(\boldsymbol{x}, t \mid \boldsymbol{x}^{\prime}, t^{\prime}\right) \tau_{\mu j}\left(\boldsymbol{x}^{\prime}, t^{\prime}\right) d \boldsymbol{x}^{\prime}, d t^{\prime}
$$

where 


$$
\begin{aligned}
\gamma_{j \mu} \equiv-(\gamma-1)\left(\frac{\partial}{\partial x_{j}^{\prime}} g_{4 \mu}-\frac{\partial \tilde{v}_{\mu}}{\partial x_{j}^{\prime}} g_{44}\right) \\
+\frac{(\gamma-1)^{2}}{2} \delta_{j \mu}\left(\frac{\partial g_{4 \ell}}{\partial x_{\ell}^{\prime}}-\frac{\partial \tilde{v}_{l}}{\partial x_{l}^{\prime}} g_{44}\right)
\end{aligned}
$$

accounts for the propagation effects and

$$
\tau_{\mu j} \equiv-\left(\rho v_{\mu}^{\prime} v_{j}^{\prime}-\bar{\rho} \widetilde{v_{\mu}^{\prime} v_{j}^{\prime}}\right)+\sigma_{\mu j}^{\prime}
$$

with

$$
v_{4}^{\prime} \equiv h_{o}^{\prime}
$$

and

$$
\sigma_{4 j}^{\prime}=-q_{j}^{\prime}+\sigma_{j \ell} v_{\ell}^{\prime}
$$

when the bulk viscosity is zero. $\tau_{v j}$, which accounts for the source structure, is just the generalized four-dimensional fluctuating Reynolds stress in the inviscid limit which is of primary interest here. Equation (28) therefore provides a direct linear relation between this quantity and the far field pressure fluctuations.

\section{Small Spread Rate Approximation}

Our interest here is in the two-dimensional shear layer with zero velocity in the upper stream. But the general solution to Eq. (26) is very complicated even for this simple case. However, high Reynolds number turbulent shear layers are nearly parallel and it is therefore appropriate to seek an asymptotic solution ${ }^{4,5}$ by expanding in the shear layer spread rate $\varepsilon$. Since the mean Reynolds stresses will certainly dominate over the viscous stresses in this case, we assume that $\bar{\sigma}_{i j}=\bar{q}_{i j}=0$.

Then the mean cross flow velocity $\tilde{v}_{3}$ and the Reynolds stresses $\tilde{T}_{\mu 3}$ and $\tilde{T}_{3 \mu}$ will vanish and the remaining mean flow variables will expand like

$$
\begin{gathered}
\tilde{v}_{1}=U\left(X, x_{2}\right)+\varepsilon U^{(1)}\left(X, x_{2}\right)+\ldots \\
\tilde{v}_{2}=\varepsilon V\left(X, x_{2}\right)+\varepsilon^{2} V^{(1)}\left(X, x_{2}\right)+\ldots \\
\bar{p}=P\left(X, x_{2}\right)+\varepsilon P^{(1)}\left(X, x_{2}\right)+\ldots \\
\bar{\rho}=\bar{R}\left(X, x_{2}\right)+\varepsilon R^{(1)}\left(X, x_{2}\right)+\ldots
\end{gathered}
$$

and

$$
\tilde{T}_{j \mu}=T_{j \mu}{ }^{(0)}+\varepsilon T_{j \mu}{ }^{(1)}+\varepsilon^{2} T_{j \mu}{ }^{(2)}+\ldots
$$

with similar expansions for $\tilde{h}, \tilde{H}_{j}$, and $\tilde{H}_{o}$. Here $X \equiv \varepsilon x_{1}$ denotes the slow streamwise variable. Substituting these into the mean flow equations and assuming that all Reynolds stresses vanish in the free streams shows that $T_{12}^{(0)}=T_{21}^{(0)}=T_{24}^{(0)}=0, P=T_{22}^{(0)}$, $P^{(1)}=T_{22}^{(1)}$ and that the lowest order mean flow equations reduce to the usual two-dimensional compressible turbulent boundary layer equations.

These results imply that the linear Euler operator $L_{\mu v}$ will expand like

$$
L_{\mu v}=L_{\mu v}^{(0)}+\varepsilon L_{\mu v}^{(1)}+\ldots
$$

and therefore that the vector Greens function $g_{v \sigma}$ has an expansion of the form

$$
g_{v \sigma}=g_{v \sigma}^{(0)}+\varepsilon g_{v \sigma}^{(1)}+\ldots
$$

where $g_{v \sigma}^{(0)}$ and $g_{v \sigma}^{(1)}$ are determined by

$$
L_{\mu v}{ }^{(0)} g_{v \sigma}{ }^{(0)}=\delta_{\mu \sigma} \delta\left(\boldsymbol{x}-\boldsymbol{x}^{\prime}\right) \delta\left(t-t^{\prime}\right)
$$

and

$$
L_{\mu \nu}{ }^{(0)} g_{\nu \sigma}{ }^{(1)}=-L_{\mu \nu}{ }^{(1)} g_{\nu \sigma}{ }^{(0)}
$$

The operators $L_{\mu \nu}^{(0)}$ and $L_{\mu \nu}^{(1)}$ are written out in detail in reference 4 . The important thing is that the left hand sides of the first 4 components of these equations are formally the same as the left sides of the Rayleigh equations (16) and since their coefficients are independent of $x_{3}$ and $t$ and depend on $x_{1}$ only through the slow streamwise variable $X$, a local (causal) solution can be constructed by taking Fourier-Laplace transforms to obtain

$$
\begin{gathered}
(\gamma-1) g_{4 \sigma}^{(0)}=-\int_{i c-\infty}^{i c+\infty} \int_{-\infty}^{\infty} \int \frac{\widetilde{c_{o}^{2}}\left(x_{2}^{\prime}\right)}{\left[k_{1} U\left(x_{2}^{\prime}\right)-\omega\right]^{2}} \\
D_{\sigma}^{\prime} e^{i\left[k_{1}\left(x_{1}-x_{1}^{\prime}\right)+k_{3}\left(x_{3}-x_{3}^{\prime}\right)-\omega\left(t-t^{\prime}\right)\right]} \bar{G}_{o}\left(x_{2} \mid x_{2}^{\prime}\right) d \omega d \boldsymbol{k}
\end{gathered}
$$


where

$$
\boldsymbol{k}=\left\{k_{1}, k_{3}\right\}
$$

is the wave number vector in the plane of the shear layer

$$
\begin{gathered}
D_{\sigma}^{\prime} \equiv \frac{\partial}{\partial x_{j}^{\prime}} \quad \text { for } \quad \sigma=j=1,2,3 \\
D_{\sigma}^{\prime} \equiv \frac{-(\gamma-1)}{\frac{c_{o}^{2}\left(x_{2}^{\prime}\right)}{2}}\left[i \omega+U\left(x_{2}^{\prime}\right) \frac{\partial}{\partial x_{1}^{\prime}}\right] \text { for } \sigma=4
\end{gathered}
$$

and the one-dimensional self adjoint Greens function $\bar{G}_{o}\left(x_{2} \mid x_{2}^{\prime}\right)$ satisfies

$$
L_{k_{1}} \bar{G}_{o}=\frac{\delta\left(x_{2}-x_{2}^{\prime}\right)}{(2 \pi)^{3}}
$$

where

$$
L_{k_{1}} \equiv \frac{\partial}{\partial x_{2}} \frac{\widetilde{c_{o}^{2}}}{\left(k_{1} U-\omega\right)^{2}} \frac{\partial}{\partial x_{2}}+1-\frac{k^{2} \widetilde{c_{o}^{2}}}{\left(k_{1} U-\omega\right)^{2}}
$$

with $k^{2}=|\boldsymbol{k}|^{2}$ is the one-dimensional reduced Rayleigh's operator.

$\bar{G}_{o}$ is therefore given by

$$
\bar{G}_{o}=\frac{w_{+}\left(x_{2}\right) w_{-}\left(x_{2}^{\prime}\right)}{\Delta(2 \pi)^{3}} \quad \text { for } x_{2}>x_{2}^{\prime}
$$

where

$$
\begin{gathered}
L_{k_{1}} w_{ \pm}=0 \\
w_{ \pm} \sim e^{ \pm \lambda_{ \pm} x_{2}} \quad x_{2} \rightarrow \pm \infty \\
\lambda_{+}=\sqrt{k^{2}-\omega^{2} / c_{+}^{2}}, \lambda_{-}=\sqrt{k^{2}-\left(k_{1} U_{-}-\omega\right)^{2} / c_{-}^{2}}
\end{gathered}
$$

$c_{+}, c_{-}$, and $U_{-}$are defined in the obvious ways and

$$
\Delta \equiv W\left(x_{2}\right) \widetilde{c_{o}^{2}}\left(x_{2}\right) /\left[k_{1} U\left(x_{2}\right)-\omega\right]^{2}
$$

where $W$ is the Wronskian of $\omega_{+}$and $\omega_{-}$is a constant independent of $x_{2}$. The result for $x_{2}<x_{2}^{\prime}$ will not be needed.

Steady state solutions can only exist if the Laplace inversion contour (shown in fig. 1) can be continuously deformed onto the real axis. But $\bar{G}^{(0)}$ possess (usually simple) poles in the upper half $k_{1}$-plane that cross the real $k_{1}$-axis during this deformation (since the shear layer is inviscidly unstable). The $k_{1}$-integration contour $c_{k_{1}}$ must therefore be deformed to lie below these poles (as shown in fig. 2) in order to obtain a continuous result ${ }^{12,13}$ The poles correspond to the eigenvalues, say $k_{1}=\kappa\left(\omega, \mathrm{k}_{3}, \mathrm{X}\right)$ of the Rayleigh operator (47). The integral over $c_{k_{1}}$ can then be decomposed into an integral over the real $k_{1}$-axis plus a contour integral that can be evaluated as the residue of the pole that crossed the real axis. The causal Greens function (43) can therefore be written as

$$
\begin{aligned}
& (\gamma-1) g_{4 \sigma}^{(0)}=-\iint_{-\infty}^{\infty} \int \frac{\widetilde{c_{o}^{2}}\left(x_{2}^{\prime}\right)}{\left[k_{1} U\left(x_{2}^{\prime}\right)-\omega\right]^{2}} \\
& D_{\sigma}^{\prime} e^{i\left[k_{1}\left(x_{1}-x_{1}^{\prime}\right)+k_{3}\left(x_{3}-x_{3}^{\prime}\right)-\omega\left(t-t^{\prime}\right)\right]} \bar{G}_{o}\left(x_{2} \mid x_{2}^{\prime}\right) d \omega d \boldsymbol{k} \\
& -2 \pi i \iiint_{-\infty}^{\infty} \int \frac{\widetilde{c_{o}^{2}}\left(x_{2}^{\prime}\right) w_{o+}\left(x_{2}\right)}{\left[\kappa U\left(x_{2}^{\prime}\right)-\omega\right]^{2} \Delta^{\prime}\left(\omega, k_{3}\right)} \\
& D_{\sigma}^{\prime} e^{i\left[\kappa\left(\omega, k_{3}\right)\left(x_{1}-x_{1}^{\prime}\right)+k_{3}\left(x_{3}-x_{3}^{\prime}\right)-\omega\left(t-t^{\prime}\right)\right]_{w_{O-}}\left(x_{2}^{\prime}\right) d \omega d k_{3}}
\end{aligned}
$$

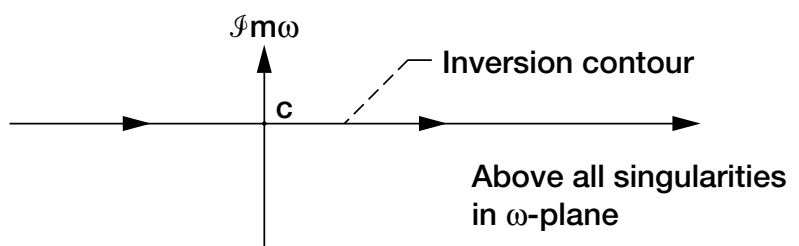

Re $\omega$

Figure 1.-Inversion contour for Laplace transform.

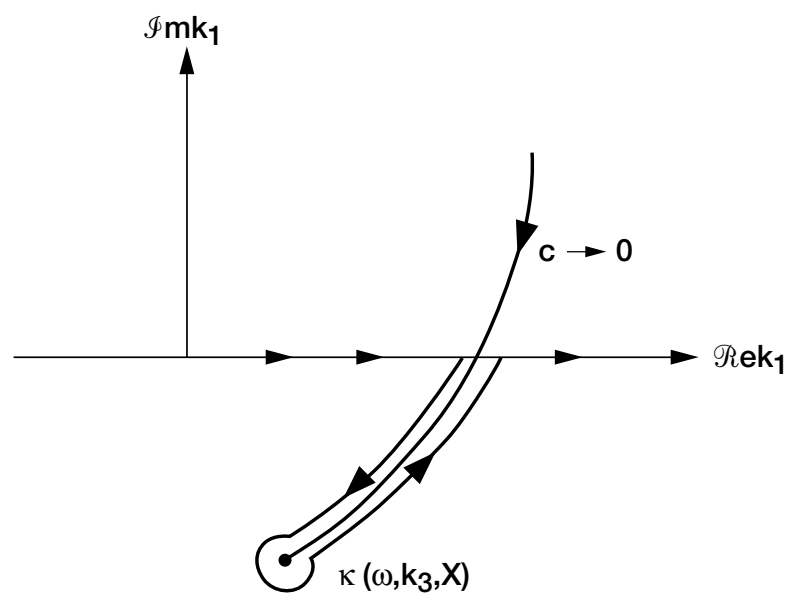

Figure 2.-Deformed $\mathrm{k}_{1}$-plane contour. 
where $\mathrm{w}_{0 \pm}$, which are now linearly dependent, are the limits of $w_{ \pm}$as $k_{1} \rightarrow \kappa$ and

$$
\Delta^{\prime} \equiv \lim _{k_{1} \rightarrow \kappa} \frac{d \Delta}{d k_{1}}
$$

The first term in this result, which corresponds to the usual Lilley's equation solutions that appear in the literature, remains bounded and is a uniformly valid approximation to the analogous term in the true nonparallel flow result. But the second terms grows without bound as $x_{1}-x_{1}^{\prime}$ becomes large (since $\left.\operatorname{Im} \kappa<0\right)$ and therefore becomes invalid on the long streamwise length scale $X-X^{\prime}$. It can, however, be rendered uniformly valid within the shear layer by using the method of multiple scales, which amounts to replacing

$$
e^{i\left[\kappa\left(\omega, k_{3}\right)\left(x_{1}-x_{1}^{\prime}\right)\right]}
$$

by

$$
A\left(X \mid X^{\prime}\right) e^{i\left[\frac{1}{\varepsilon} \int_{X^{\prime}}^{X} \kappa\left(\omega, k_{3}, X\right) d X\right]}
$$

where the slowly varying amplitude function $A$, which satisfies the initial condition

$$
A\left(X \mid X^{\prime}\right) \rightarrow 1 \text { as } X \rightarrow X^{\prime}
$$

is determined by imposing a solvability condition on the next order solution. The detailed results are given in reference 4 .

But, this solution is still not uniformly valid at large transverse distances where $Y \equiv \varepsilon x_{2}=O(1)$, because the second order solution ${ }^{14}$ (which is a generalization of the second order instability wave solution considered by Tam and Morris) now decays more slowly with $x_{2}$ as $x_{2} \rightarrow \pm \infty$ than the first order solution. But an "outer" solution can be constructed by using the WKBJ method to solve the reduced stationary (or uniformly moving) media wave equation that governs the flow in that region This result can be used to form a "composite" solution ${ }^{17}$ that remains uniformly valid everywhere within and outside the shear layer. The details are given in reference 4. Our interest here is in the upper region and we therefore do not require uniformity below the shear layer.

The final uniformly valid Greens function $g_{4 \sigma}$ can be written as

$$
(\gamma-1) g_{4 \sigma}^{(0)}=\int_{-\infty}^{\infty} e^{-i \omega\left(t-t^{\prime}\right)} G_{4 \sigma}^{(0)}\left(\boldsymbol{x} \mid \boldsymbol{x}^{\prime} ; \omega\right) d \omega
$$

where

$$
\begin{aligned}
& G_{4 \sigma}^{(0)}=-\int_{-\infty}^{\infty} \int \frac{\widetilde{c_{o}^{2}}\left(x_{2}^{\prime}\right)}{\left[k_{1} U\left(x_{2}^{\prime}\right)-\omega\right]^{2}} \\
& D_{\sigma}^{\prime} e^{i\left[k_{1}\left(x_{1}-x_{1}^{\prime}\right)+k_{3}\left(x_{3}-x_{3}^{\prime}\right)\right]} \bar{G}_{o}\left(x_{2} \mid x_{2}^{\prime}\right) d \boldsymbol{k} \\
& -\frac{i}{(2 \pi)^{2}} \int_{-\infty}^{\infty} \frac{c_{o}^{2}\left(x_{2}^{\prime}\right) A\left(X_{o} \mid X^{\prime}\right) w_{o+}\left(x_{2}\right)}{\left[\kappa U\left(x_{2}^{\prime}\right)-\omega\right]^{2} \Delta^{\prime}\left(\omega, k_{3}\right) \sqrt{1+\alpha^{\prime}\left(X_{o}\right) Y}} \\
& D_{\sigma}^{\prime} e^{i\left[\frac{1}{\varepsilon} \bar{\Theta}(X, Y)+k_{3}\left(x_{3}-x_{3}^{\prime}\right)\right]} w_{w^{-}}\left(x_{2}^{\prime}\right) d k_{3} \\
& \bar{\Theta} \equiv \Theta-i \sqrt{\kappa^{2}(X)+k_{3}^{2}-\left(\omega / c_{+}\right)^{2}} Y \\
& \Theta=\int_{X^{\prime}}^{X_{0}} \kappa(Z) d Z-\frac{i\left[\left(\omega / c_{+}\right)^{2}-k_{3}^{2}\right] Y}{\sqrt{\kappa^{2}\left(X_{0}\right)+k_{3}^{2}-\left(\omega / c_{+}\right)^{2}}} \\
& X_{o} \equiv X-\alpha\left(X_{0}\right) Y \\
& \alpha \equiv \frac{\kappa}{i \sqrt{\kappa^{2}+k_{3}^{2}-\left(\omega / c_{+}\right)^{2}}}
\end{aligned}
$$

with the arguments $k_{3}$ and $\omega$ suppressed. The functions $\bar{G}_{o}$ and $\Delta^{\prime}$ are defined by Eqs. (48), (52), and (54).

It is necessary to evaluate Eq. (57) in the region where

$$
R^{2}=\varepsilon^{2} r^{2} \rightarrow \infty
$$

with

$$
r^{2} \equiv \sqrt{x_{1}^{2}+x_{2}^{2}+x_{3}^{2}}
$$

in order to in calculate the radiated sound at large distances from the shear layer. But applying the method of stationary phase and using Eqs. (48), (50), (51) along the asymptotic behavior of $\Theta$ and $a$ shows that

$$
\begin{aligned}
G_{4 \sigma}^{(0)} \rightarrow-\frac{e^{i \omega r / c_{+}}}{(2 \pi)^{2} r} \frac{\omega}{c_{+}} \sin \theta \cos \varphi \\
\\
\quad\left[G_{B \sigma}\left(\hat{\boldsymbol{x}} \mid \boldsymbol{x}^{\prime}\right)+G_{I \sigma}\left(\hat{\boldsymbol{x}} \mid \boldsymbol{x}^{\prime}\right)\right]
\end{aligned}
$$


when the azimuthal polar angle $\theta$ and the Mach number $M \quad$ are sufficiently large. The $G_{B \sigma}\left(\hat{\boldsymbol{x}} \mid \boldsymbol{x}^{\prime}\right)$ and $G_{I \sigma}\left(\hat{\boldsymbol{x}} \mid \boldsymbol{x}^{\prime}\right)$ are defined in reference $4, \quad \varphi$ is the circumferential polar angle and $\hat{\boldsymbol{x}}=\hat{\boldsymbol{x}}(\theta, \varphi)$ denotes the unit vector in the $\boldsymbol{x}$-direction.

\section{The Far Field Acoustic Spectrum}

The quantity of interest is the mean square acoustic pressure

$$
\overline{p^{2}} \equiv \frac{1}{2 T} \int_{-T}^{T} p_{o}^{\prime}(\boldsymbol{x}, t) p_{o}^{\prime}\left(\boldsymbol{x}, t+t_{o}\right) d t
$$

at large distances above the shear layer, where we have used the fact that $p_{o}^{\prime} \rightarrow p^{\prime}$ in this region (see Eqs. (11), (23), and (24)) and $T$ denotes a large but finite time interval. Substituting Eq. (28) into this result and changing integration variables shows that

$$
\begin{array}{r}
\overline{p^{2}}\left(\boldsymbol{x}, t_{o}\right)=\int_{-\infty}^{\infty} \int_{V} \int_{\gamma} \bar{\gamma}_{j \sigma \mu \ell}\left(\boldsymbol{x} \mid \boldsymbol{x}^{\prime} ; \boldsymbol{\zeta}, t_{o}+\tau\right) \\
\bar{\tau}_{\sigma j \mu \ell}\left(\boldsymbol{x}^{\prime} ; \boldsymbol{\zeta}, \tau\right) d \boldsymbol{x}^{\prime} d \boldsymbol{\zeta} d \tau
\end{array}
$$

where

$$
\bar{\gamma}_{j \sigma \ell \mu} \equiv \int_{-\infty}^{\infty} \gamma_{j \sigma}\left(\boldsymbol{x} \mid \boldsymbol{x}^{\prime}, t_{1}+t_{o}+\tau\right) \gamma_{\ell \mu}\left(\boldsymbol{x} \mid \boldsymbol{x}^{\prime}+\boldsymbol{\zeta}, t_{1}\right) d t_{1}
$$

accounts for the acoustic propagation and mean flow interaction effects and

$$
\bar{\tau}_{\sigma i \mu j} \equiv \frac{1}{2 T} \int_{-T}^{T} \tau_{\sigma i}\left(\boldsymbol{x}^{\prime}, t^{\prime}\right) \tau_{\mu j}\left(\boldsymbol{x}^{\prime}+\zeta, t^{\prime}+\tau\right) d t^{\prime}
$$

is the density weighted fourth order 2-point time delayed turbulent velocity/total enthalpy correlation.

Equation (66) provides a direct linear relation between the acoustic pressure and the fourth order correlations of the turbulent fluctuations in the shear layer. Unfortunately, the latter quantity is very difficult to determine - either experimentally or numerically - and it is, therefore, highly desirable to make the acoustic predictions as insensitive to its details as possible. It would be possible to make considerable progress toward achieving that objective if the propagation factor, $\bar{\gamma}_{j \sigma \mu \ell}$, were to vary only slowly over the correlation length of $\bar{\tau}_{\sigma j \mu \ell}$, because the former could then be treated as a constant relative to the $\zeta$-integration and the result would then depend only on the temporal correlation of the turbulence at any given point in the shear layer. This is probably the true in the cross stream direction, but $\bar{\tau}_{\sigma j \mu \ell}$ is expected to decay much more slowly in the stream-wise directionespecially at the higher Mach numbers of technological interest. However, Lighthill ${ }^{15}$ pointed out that the $\varsigma_{1}$-decay should be much more rapid in a reference frame moving with the convection velocity, $U_{c}$, of the turbulence and Ffowcs Williams ${ }^{16}$ showed that this idea can best be implemented by introducing the moving frame correlation

$$
\tau_{\sigma j \mu \ell}^{M}\left(\boldsymbol{x}^{\prime} ; \boldsymbol{\xi}, \tau\right) \equiv \bar{\tau}_{\sigma j \mu \ell}\left(\boldsymbol{x}^{\prime} ; \boldsymbol{\zeta}, \tau\right)
$$

where

$$
\xi \equiv \zeta-\hat{i} U_{c} \tau
$$

into Eq. (66) and changing the integration variable from $\zeta$ to $\xi$.

The result, which is much simpler when re-expressed in terms of the acoustic spectrum

$$
I_{\omega}(\boldsymbol{x})=\frac{1}{2 \pi} \int_{-\infty}^{\infty} e^{i \omega t_{o}} \overline{p^{2}}\left(\boldsymbol{x}, t_{o}\right) d t_{o}
$$

or better yet in terms of $I_{\omega}\left(\boldsymbol{x} \mid \boldsymbol{x}^{\prime}\right)$

$$
I_{\omega}(\boldsymbol{x})=\int_{V} I_{\omega}\left(\boldsymbol{x} \mid \boldsymbol{x}^{\prime}\right) d \boldsymbol{x}^{\prime}
$$

the acoustic spectrum at $\boldsymbol{x}$ due to a unit volume of turbulence at $\boldsymbol{x}^{\prime}$,is given by

$$
\begin{aligned}
& I_{\omega}\left(\boldsymbol{x} \mid \boldsymbol{x}^{\prime}\right)= \\
& 2 \pi \int_{-\infty}^{\infty} \int_{V} \Gamma_{j \sigma}\left(\mathbf{x} \mid \boldsymbol{x}^{\prime} ; \omega\right) \Gamma_{\ell \mu}^{*}\left(\mathbf{x} \mid \boldsymbol{x}^{\prime}+\boldsymbol{\xi}+\hat{\boldsymbol{i}} U_{c} \tau ; \omega\right) \\
& e^{-i \omega \tau} \tau_{\sigma j \mu \ell}^{M}\left(\boldsymbol{x}^{\prime}, \boldsymbol{\xi}, \tau\right) d \boldsymbol{\xi} d \tau
\end{aligned}
$$

where capital letters denote Fourier transforms (with respect to time) of the corresponding lower case letters, and the asterisk denotes complex conjugates.

When $\theta$ and $M$ are sufficiently large, equation (64) and the form of $G_{B \mu}$ and $G_{I \mu}$ given in reference 4 imply that

$$
\begin{aligned}
\Gamma_{j \sigma}=-\frac{e^{i \omega r / c_{+}} \omega}{(2 \pi)^{2} c_{+} r} \sin \theta \cos \varphi & \\
& {\left[\Gamma_{B j \sigma}\left(\hat{\boldsymbol{x}} \mid \boldsymbol{x}^{\prime}\right)+\Gamma_{I j \sigma}\left(\hat{\boldsymbol{x}} \mid \boldsymbol{x}^{\prime}\right)\right] }
\end{aligned}
$$


where

$$
\begin{aligned}
& \Gamma_{B j \sigma} \equiv \tilde{\Gamma}_{B j \sigma}\left(\hat{\boldsymbol{x}} \mid \boldsymbol{x}_{\perp}^{\prime}\right) e^{-i \frac{\omega}{c_{+}} x_{1} \cos \theta}=\tilde{D}_{j \sigma \mu} G_{B \mu}\left(\hat{\boldsymbol{x}} \mid \boldsymbol{x}^{\prime}\right) \\
& \Gamma_{I j \sigma} \equiv \tilde{\Gamma}_{I j \sigma}\left(\hat{\boldsymbol{x}} \mid \boldsymbol{x}_{\perp}^{\prime}\right) e^{\frac{i}{\varepsilon} \Theta_{\infty}\left(\theta, \varphi, X^{\prime}\right)}=\tilde{D}_{j \sigma \mu} G_{I \mu}\left(\hat{\boldsymbol{x}} \mid \boldsymbol{x}_{\perp}^{\prime}\right) \\
& \Theta_{\infty} \equiv \int_{X^{\prime}}^{\bar{\alpha}_{0}} \kappa(Z) d Z-\frac{\omega \bar{\alpha}_{0}}{c_{+}} \sqrt{1-\sin ^{2} \theta \sin ^{2} \varphi} \cos \theta
\end{aligned}
$$

$\bar{\alpha}_{0}$ is determined implicitly by

$$
\begin{gathered}
\kappa\left(\omega, \frac{\omega}{c_{+}} \sin \theta \sin \varphi, \bar{\alpha}_{0}\right)=\frac{\omega}{c_{+}} \cos \theta \\
\tilde{D}_{j \sigma \mu} \equiv-\delta_{\sigma \mu} \frac{\partial}{\partial x_{j}^{\prime}}+\frac{\partial U}{\partial x_{j}^{\prime}} \delta_{\sigma 1} \delta_{\mu \sigma}+\frac{(\gamma-1)}{2} \delta_{j \sigma} \frac{\partial}{\partial x_{\mu}^{\prime}}
\end{gathered}
$$

and $\boldsymbol{x}_{\perp}^{\prime}=\left\{x_{2}^{\prime}, x_{3}^{\prime}\right\}$ is the cross flow source variable.

Since $\Theta_{\infty}$ varies on the slow streamwise length scale $\boldsymbol{X}^{\prime}$, which is much longer than the correlation length of the turbulence, we can account for its variation over the range of integration in Eq. (73) by expanding it in a Taylor series to show that

$$
\begin{aligned}
\Theta_{\infty}\left(\hat{\mathbf{x}}, X^{\prime}+\varepsilon\left(\xi_{1}+U_{c} \tau\right)\right) & +\Theta_{\infty}\left(\hat{\mathbf{x}}, X^{\prime}\right) \\
& -\varepsilon \kappa\left(X^{\prime}\right)\left(\xi_{1}+U_{c} \tau\right)
\end{aligned}
$$

with more than enough accuracy to evaluate this result. Using this in Eq. (76) and inserting the result along with Eqs. (74) and (75) into Eq. (73) shows that

$$
\begin{aligned}
& I_{\omega}\left(\boldsymbol{x} \mid \boldsymbol{x}^{\prime}\right)=\left(\frac{\omega \sin \theta \cos \varphi}{c_{+} r 2 \pi}\right)^{2} \\
& \int_{V}\left[\Gamma_{B j \sigma}\left(\hat{\boldsymbol{x}} \mid \boldsymbol{x}^{\prime}\right)+\Gamma_{I j \sigma}\left(\hat{\boldsymbol{x}} \mid \boldsymbol{x}^{\prime}\right)\right] \\
& {\left[\Gamma_{B \ell \mu}^{*}\left(\hat{\boldsymbol{x}} \mid \boldsymbol{x}^{\prime}+\boldsymbol{\xi}\right) H_{\sigma j \mu \ell}^{*}\left(\boldsymbol{x}^{\prime} ; \boldsymbol{\xi}, \omega\left(1-M_{c} \cos \theta\right)\right)\right.} \\
& \left.+\Gamma_{I \ell \mu}^{*}\left(\hat{\boldsymbol{x}} \mid \boldsymbol{x}^{\prime}+\boldsymbol{\xi}\right) H_{\sigma j \mu \ell}^{*}\left(\boldsymbol{x}^{\prime} ; \boldsymbol{\xi}, \omega-U_{c} \boldsymbol{\kappa}^{*}\left(X^{\prime}, \omega\right)\right)\right] d \boldsymbol{\xi}
\end{aligned}
$$

where

$$
H_{\sigma j \mu \ell}^{*}\left(\boldsymbol{x}^{\prime} ; \boldsymbol{\xi}, \omega\right) \equiv \frac{1}{2 \pi} \int_{-\infty}^{\infty} e^{i \omega \tau} \tau_{\sigma j \mu \ell}^{M}\left(\boldsymbol{x}^{\prime} ; \boldsymbol{\xi}, \tau\right) d \tau
$$

denotes the 2-point fourth order turbulence spectrum relative to the moving frame and the second terms in the square brackets are identically zero when $\omega$ is greater than the neutral frequency where $\operatorname{Im} \kappa\left(\omega x_{o}^{\prime}\right)=0$.

This result is nearly exact. The only significant approximation is that the mean flow spread rate, $\varepsilon$, is small. But $H_{\text {бjue }}$ is expected to vary much more rapidly with $\xi$ than $\Gamma_{e \mu}$ and Eq. (80) can therefore be approximated by

$$
\begin{aligned}
& I_{\omega}\left(\boldsymbol{x} \mid \boldsymbol{x}^{\prime}\right)=\left(\frac{\omega \cos \varphi \sin \theta}{c_{+} r 2 \pi}\right)^{2} \\
& {\left[\Gamma_{B j \sigma}\left(\hat{\boldsymbol{x}} \mid \boldsymbol{x}^{\prime}\right)+\Gamma_{I j \sigma}\left(\hat{\boldsymbol{x}} \mid \boldsymbol{x}^{\prime}\right)\right]} \\
& {\left[\Gamma_{B \ell \mu}^{*}\left(\hat{\boldsymbol{x}} \mid \boldsymbol{x}^{\prime}\right) \Phi_{\sigma j \mu \ell}^{*}\left(\boldsymbol{x}^{\prime} ; \omega\left(1-M_{c} \cos \theta\right)\right)\right.} \\
& \left.+\Gamma_{I \ell \mu}^{*}\left(\hat{\boldsymbol{x}} \mid \boldsymbol{x}^{\prime}\right) \Phi_{\sigma j \mu \ell}^{*}\left(\boldsymbol{x}^{\prime} ; \omega-U_{c} \kappa^{*}\left(\omega, X^{\prime}\right)\right)\right]
\end{aligned}
$$

where

$$
\Phi_{\sigma j \mu \ell}\left(\boldsymbol{x}^{\prime} ; \omega\right) \equiv \int_{V} H_{\sigma j \mu \ell}\left(\boldsymbol{x}^{\prime} ; \boldsymbol{\xi}, \omega\right) d \boldsymbol{\xi}
$$

is the single point fourth order turbulence spectral tensor at $\boldsymbol{x}^{\prime}$.

This approximation cannot remain valid when $M_{c} \cos \theta=1$ because the first spectral function in Eq. (82) does not $\rightarrow 0$ as $\omega \rightarrow \infty$ in this case, which causes the integral of Eq. (82) with respect to $\omega$ (and therefore the mean square pressure) to become infinite. Ffowcs Williams ${ }^{16}$ argued that this type of difficulty could be overcome by replacing the Doppler factor $\left(1-M_{c} \cos \theta\right)$ with

$$
1-M_{c} \cos \theta \rightarrow \sqrt{\left(1-M_{c} \cos \theta\right)^{2}+\left(a M_{c}\right)^{2}}
$$

where $a$ is a small constant. While this result is not strictly valid in the present context, it may be useful for obtaining numerical results.

Notice that Eq. (82) can now be evaluated without performing any quadritures once the appropriate solutions to the Rayleigh equation (49) have been found. But this equation is still fairly complex and a great deal of modeling and/or computation is required to evaluate all of its terms. It is therefore worthwhile to 
introduce some approximate results that can be used to get some insight into the import physical effects.

\section{Some Approximate Results}

It is well known ${ }^{20}$ that the Reynolds stresses $T_{11}^{(0)}$ and $T_{12}^{(1)}$ that appear in the lowest order mean flow equations can be chosen so that these equations possess similarity solutions of the form

$$
U=U(\eta), \bar{R}=\bar{R}(\eta)
$$

where

$$
\eta \equiv x_{2} / x
$$

and since this causes no particular inconsistency with the expansions (33) through (37) and agrees well with experimental observation we shall now assume this to be the case.

We also suppose that the observation point lies in the plane perpendicular to that of the shear layer, i.e. that the azimuthal angle $\varphi=0$. Then we can evaluate all of the factors in Eq. (82) by solving the one-dimensional Rayleigh equation, i.e. by setting $k_{3}=0$ (or equivalently $k=k_{1}$ ) in Eq. (47) which now becomes

$$
L_{k_{1}} \rightarrow \frac{d}{d \eta} \frac{\widetilde{c_{o}^{2}}}{(\sigma U-1)^{2}} \frac{d}{d \eta}+\bar{\omega}^{2}\left[1-\frac{\widetilde{\sigma^{2} c_{o}^{2}}}{(\sigma U-1)^{2}}\right]
$$

where

$$
\bar{\omega} \equiv X \omega
$$

and

$$
\sigma \equiv k_{1} / \omega
$$

is the reciprocal of the complex phase speed. The eigenvalue $\kappa$ in Eq. (76) can now be replaced by

$$
\sigma_{o}(\bar{\omega}) \equiv \frac{\kappa}{\omega}
$$

i.e. it is a function of the single variable $\bar{\omega}$ which means that $\bar{\alpha}_{o}$ is now determined by

$$
\sigma_{o}(\bar{\Omega})=\frac{1}{c_{+}} \cos \theta
$$

where $\bar{\Omega} \equiv \omega \bar{\alpha}_{o}$ is essentially the complex frequency eigenvalue of the Rayleigh problem corresponding to the specified real phase speed $c_{+} / \cos \theta$.

Additional simplification can be achieved by assuming that

$$
\Phi_{\sigma j \mu \ell}(\omega) \simeq \delta_{\sigma j} \delta_{\mu \ell} \Phi_{o}(\omega)
$$

which implies, among other things, that $h_{o}^{\prime} \simeq 0$.For simplicity we also neglect the cross coupling between the two components of the Greens function and set $\widetilde{c_{o}^{2}}$ $=c_{+}^{2}=$ constant. It then follows from Eqs. (45), (47), (65) , (74) through (77) and (82) that

$I_{\omega}\left(\boldsymbol{x} \mid \boldsymbol{x}^{\prime}\right)=\left[\frac{\omega^{3} X^{\prime}(\gamma+1)}{2 \pi c_{+}^{3} r}\right]^{2}\left\{\left|\frac{w_{-}\left(\eta^{\prime}, \bar{\omega}, \frac{1}{c_{+}} \cos \theta\right)}{\bar{\Delta}\left(\bar{\omega}, \frac{1}{c_{+}} \cos \theta\right)}\right|^{2}\right.$

$\Phi_{o}\left(\omega\left(1-M_{o} \cos \theta\right)\right)+\left|\frac{w_{o-}\left(\eta^{\prime}, \bar{\omega}\right) A\left(\bar{\Omega} \mid X^{\prime}\right)}{\bar{\Delta}^{\prime}(\bar{\omega})}\right|^{2}$

$\left.\frac{2 \pi}{\varepsilon \sigma_{o}^{\prime}} e^{-(2 / \varepsilon) \operatorname{Im} \Theta_{\infty}(\theta, \bar{\omega})} \operatorname{Re} \Phi_{o}\left(\omega\left(1-U_{c} \sigma_{o}(\bar{\omega})\right)\right)\right\}$

where

$$
\begin{gathered}
\bar{\Delta} \equiv \frac{W_{o}}{(\sigma U-1)^{2}} \\
\bar{\Delta}^{\prime} \equiv \lim _{\sigma \rightarrow \sigma_{o}} \frac{\partial \bar{\Delta}}{\partial \sigma} \\
\sigma_{o}^{\prime} \equiv \frac{d \sigma_{o}}{d \bar{\Omega}}
\end{gathered}
$$

and $W_{o}$ denotes the Jacobian with derivatives with respect to $\eta$.

\section{$\underline{\text { Numerical Results }}$}

To compute the radiated sound field from Eq. (93) it is necessary to specify the turbulence spectra $\Phi_{o}$. Recent numerical studies ${ }^{18,20}$ suggest that it should be of the Gaussian form

$$
\Phi_{o}=\frac{2 \pi\left(\rho_{s} u_{s}^{2}\right)^{2} a_{s}}{\omega_{s}} \ell_{s}^{3} e^{-\left(\omega / 2 \omega_{s}\right)^{2}}
$$

where $\bar{\omega}_{s}=X^{\prime} \omega_{s}, \quad \bar{\ell}_{s}=\ell_{s} / X^{\prime}, \rho_{s}$, and $U_{s}$ are constants with obvious physical interpretation and $a_{s}$ is an empirically determined scale factor. It is also necessary to specify the mean velocity profile $U(\eta)$ in the shear layer which is reasonably well represented by $^{20}$

$$
U=\frac{1}{2}(1-\tanh \eta)
$$


The result is, as it should be, independent of the axial source location $X^{\prime}$ within the shear layer when expressed in terms of the scaled frequency $\bar{\omega} \equiv X^{\prime} \omega$.

When $\operatorname{Im} \Theta_{\infty}=\mathrm{O}(1)$, the $1 / \varepsilon$ factor in the exponent of Eq. (93) will cause the second term to be negligibly small when $\operatorname{Im} \Theta_{\infty}>0$ and very large (i.e. it will be dominant) when $\operatorname{Im} \Theta_{\infty}<0$. The $\varepsilon$ in the denominator will cause his term to be large when $\operatorname{Im} \Theta_{\infty}=\mathrm{O}(\varepsilon)$ no matter what its sign .

Figure 3 is a plot of $\operatorname{Im} \Theta_{\infty}$ as a function of $\theta$ for various values of the Mach number $M$ and source frequency $\bar{\omega}$. The curves are truncated at small $\theta$ because the second term has no radiation field beyond this point, i.e. it becomes evanescent there. These results show that $\operatorname{Im} \Theta_{\infty}$ is always positive at $\theta=90^{\circ}$ but can change sign and become negative when $M$ is sufficiently large and $\theta$ is sufficiently small.

The spectral shape at $90^{\circ}$ is therefore produced by the first term in Eq. (93) which is primarily determined by the factor

$$
\left(\frac{\omega}{c_{\infty}}\right)^{6} \Phi_{o}\left(\omega\left(1-M_{c} \cos \theta\right)\right)=\left(\frac{\omega}{c_{\infty}}\right)^{6} \Phi_{o}(\omega)
$$

at that angle. Refraction effects, which are accounted for by the first factor in this term, will cut off the high part of the source spectrum at small angles to the axis. But figure 3 shows that the second term can become dominant at these angles when $M$ is sufficiently large. Its spectral shape, which is primarily determined by

$$
\left(\frac{\omega}{c_{\infty}}\right)^{6} \operatorname{Re} \Phi_{o}\left(\omega-U_{c} \kappa\left(X^{\prime}, \omega\right)\right) H\left(-\operatorname{Im} \kappa\left(X^{\prime}, \omega\right)\right)
$$

where $H(x)$ denotes the Heaviside function $H(x)=1,0$ $x \gtrless 0$, is relatively independent of angle and has a much narrower width than $\Phi_{o}(\omega)$ due to the relatively narrow band of unstable frequencies at $X^{\prime}$.

\section{Concluding Remarks}

The Navier-Stokes equations, rewritten in the form of the LNS equations with externally applied stress and energy flux sources were solved for a two-dimensional shear layer by using a vector Greens function approach and assuming that the spread rate $\varepsilon$ of the shear layer is small. The relevant Greens function has two components -one of which corresponds to the usual Lilley equation solutions that appear in the literature and the other associated with linear instability waves.

Numerical results obtained for a simplified source model show encouraging qualitative agreement with experimental observations. Observed differences in spectral shapes at small and large angles to the downstream axis that occur at the higher Mach numbers can, in part, be attributed to the fact that the first Greens function component is dominant at $\theta=90^{\circ}$ while the second component is dominant at small $\theta$. 

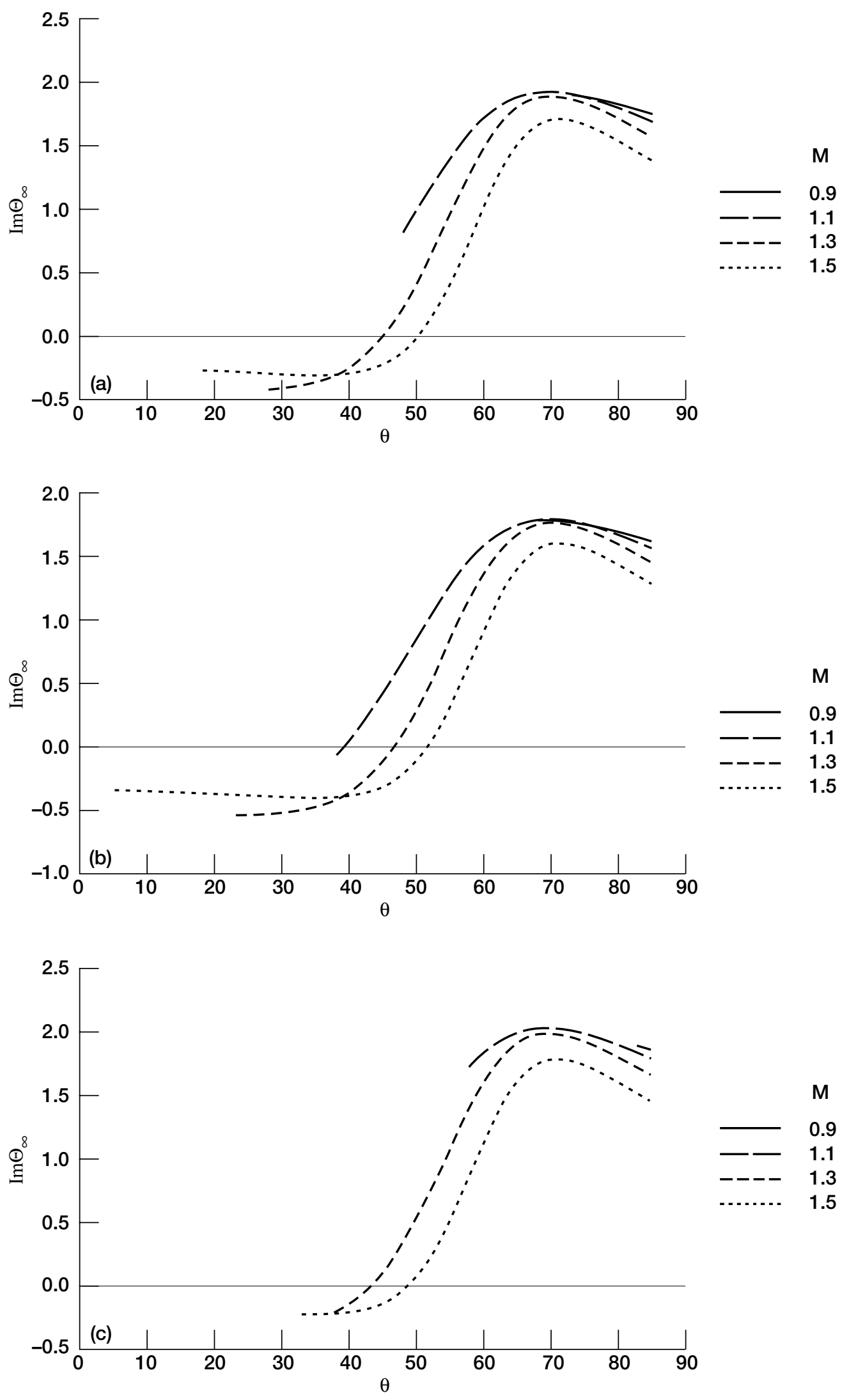

Figure 3. $-\operatorname{Im} \Theta_{\infty}(\theta, \omega)$ vs $\theta$. (a) $\bar{\omega}=0.2$. (b) $\bar{\omega}=0.1$. (c) $\bar{\omega}=0.3$. 


\section{$\underline{\text { References }}$}

1. Balsa, T.F., Gliebe, P.R., Kantola, R.A., Mani, R., Strings, E.J., and Wong, J.C.F., II (1978) High velocity jet noise source location and reduction, FAA Report No. FAA-RD-76-79.

2. Lilley, G.M. (1974) On the noise from jets, Noise Mechanism, AGARD-CP-131, pp. 13.1-13.12.

3. Dowling, A.P., Ffowcs Williams, J.E., and Goldstein, M.E. (1978) Sound propagation in a moving stream, Phil. Trans. Roy. Soc. Lond., A, vol. 288, no. 1353, pp. 321-349.

4. Goldstein, M.E. and Leib, S.J. (2003) A Causal Approach to Predicting Jet Noise. To be submitted, J. Fl. Mech.

5. Goldstein, M.E. (2003) A Generalized Acoustic Analogy. To be published, J. Fl. Mech.

6. Betchov, R. and Criminale, W.O. (1967) Stability of parallel flows, Academic Press.

7. Goldstein, M.E. (1976) Aeroacoustics, McGrawHill.

8. Lele, S.K. (1994) Compressibility effects in turbulence, Annual Rev. of Fluid Mech., vol. 26, pp. 211-254.

9. Speziale, C.G. (1991) Analytical methods for the development of Reynolds-stress closure in turbulence, Ann. Rev. Fluid Mech., vol. 23, pp. 107-157.

10. Speziale, C.G. and So, M.C. (1998) Turbulence modeling an simulation in the Handbook of Fluid Dynamics, Richard Johnson, ed., CRC Press.
11. Morse, P.M. and Feshbach, H. (1953) Methods of theoretical physics, McGraw-Hill, pp. 878-886.

12. Briggs, R.J. 1964 Electron Stream Interaction with Plasmas. Massachusetts Institute of Technology Press.

13. Bers, A. 1975 Linear waves and instabilities. Plasma Physics (ed. C.DeWitt and J. Perraud), pp. 113-2166 Gordon \& Beach.

14. Tam, C.K.W. and Morris, P.J.(1980) The radiation of sound by instability waves of a compressible plane turbulent mixing layer, J. of Fluid Mech., 98, pp. 349-381.

15. Lighthill, M.J. (1952) On sound generated aerodynamically: I. General theory, Proc. R. Soc. Lond., A211, pp. 564-587.

16. Ffowcs Williams, J.E. (1963) The Noise From Turbulence Converted at High Speed, Phil. Trans. Roy. Soc. A225, pp. 469-503.

17. Van Dyke, M. (1975) Perturbation Methods in Fluid Mechanics, The Parabolic Press.

18. Freund, J.B. (2002) Turbulent Jet Noise: Shear Noise, Self-Noise and Other Contributions, AIAA paper 2002-2423.

19. Goldstein, M.E. (2002) A unified approach to some recent developments in jet noise theory, International Journal of Aeroacoustics, vol. 1, no. 1, pp. 1-16.

20. Pope, S.B. (2000) Turbulent Flows, Cambridge University Press. 



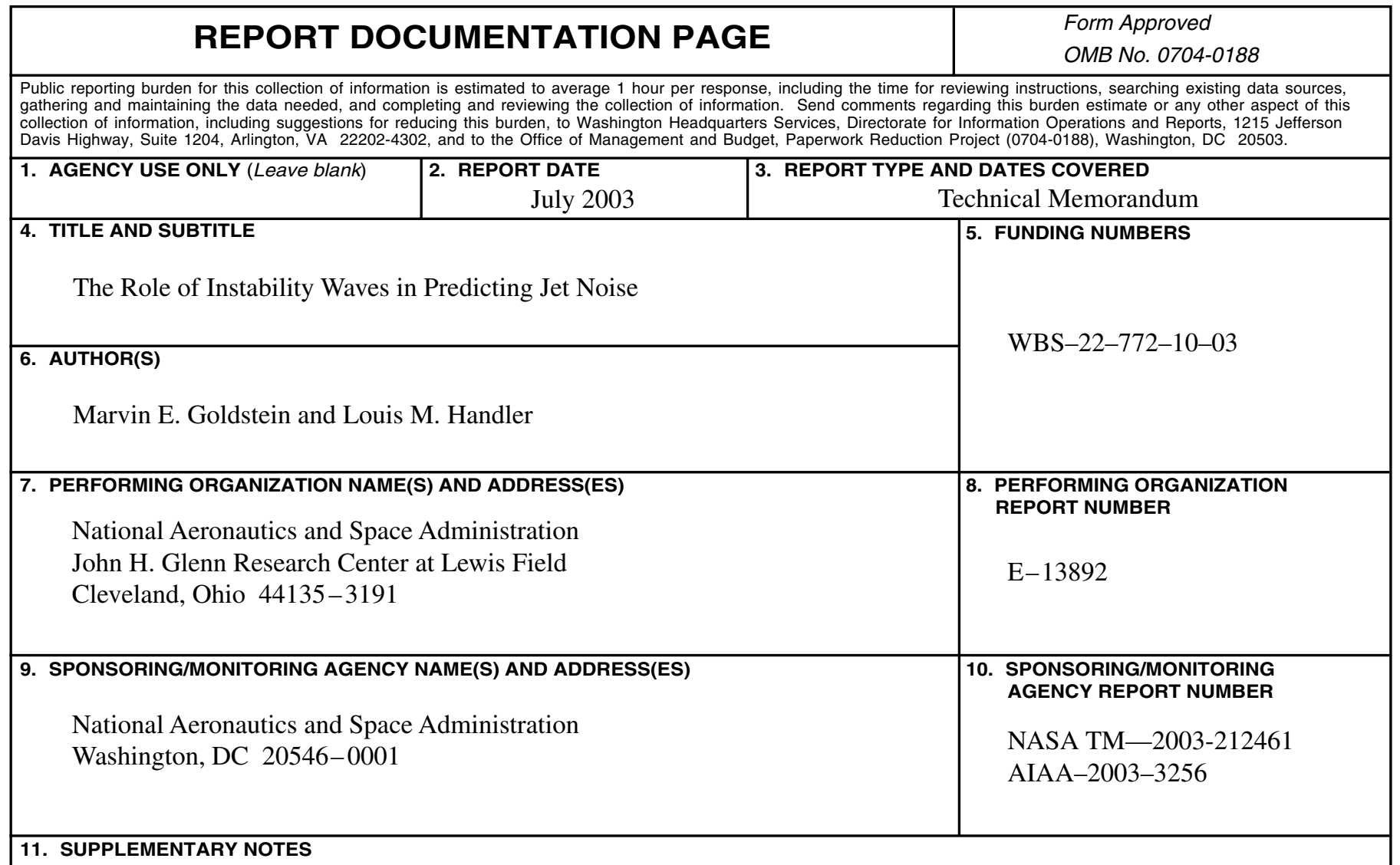

Prepared for the Ninth Aeroacoustics Conference and Exhibit cosponsored by the American Institute of Aeronautics and Astronautics and the Confederation of European Aerospace Societies, Hilton Head, South Carolina, May 12-14, 2003. Responsible person, Marvin E. Goldstein, organization code 0100, 216-433-5825.

12a. DISTRIBUTION/AVAILABILITY STATEMENT

12b. DISTRIBUTION CODE

Unclassified - Unlimited

Subject Categories: 02 and 71

Distribution: Nonstandard

Available electronically at http://gltrs.grc.nasa.gov

This publication is available from the NASA Center for AeroSpace Information, 301-621-0390.

13. ABSTRACT (Maximum 200 words)

Debate over whether linear instability waves play a role in the prediction of jet noise has been going on for many years. Parallel mean flow models, such as the one proposed by Lilley, usually neglect these waves because they cause the solution to become infinite. The present paper solves the true non-parallel acoustic equations for a two-dimensional shear layer by using a vector Greens function and assuming small mean flow spread rate. The results show that linear instability waves must be accounted for in order to construct a proper causal solution to the problem.

\begin{tabular}{|c|c|c|c|}
\hline \multicolumn{3}{|l|}{ 14. SUBJECT TERMS } & $\begin{array}{c}\text { 15. NUMBER OF PAGES } \\
18\end{array}$ \\
\hline NSN 7540-01-280-5500 & & & $\begin{array}{l}\text { andard Form } 298 \text { (Rev. 2-89) } \\
\text { scribed by ANSI Std. Z39-18 } \\
-102\end{array}$ \\
\hline
\end{tabular}

